\title{
METAL-FORMING STUDIES BY MOIRÉ INTERFEROMETRY
}

\author{
by S.D.K. Barbat, Y. Guo, R.S. Rao and D. Post
}

Studies of metal-forming processes are hampered by the uncertainty of boundary conditions at the interface between the metal and the dies (i.e., the forming tools). In the investigation reviewed here, the boundary conditions were determined experimentally for a case of strip drawing. Most previous experimental analyses of the cold-drawing process used photoelasticity with plastic models of die and workpiece materials. Some studies utilized metal workpiece materials and transparent sapphire dies, ${ }^{1.2}$ which provided visual access to the interfacial conditions. On the other hand, the current research is directed toward the study of industrial conditions, using hardened tool-steel dies and steel workpiece material.

The drawing process used in this experiment is illustrated in Fig. 1, where low-carbon steel strips are drawn through hardened steel dies to reduce its thickness. During the steady-state drawing process, the deformation of one die was measured by high-sensitivity moiré interferometry. ${ }^{3}$ This deformation was analyzed to determine the normal and shear strains along the contact surface of the die. Since the elastic properties of the die material were known, the corresponding contact stresses (both normal and shear stresses) were readily calculated. These shear stresses were generated by frictional forces alone, and therefore, they enabled the determination of the interface friction conditions.

The preliminary test reported here is intended to verify the merits of the experimental approach and to suggest refinements for future tests. The results showed unexpected and fascinating characteristics of the unlubricated drawing process. While the measurements appear to give compelling evidence for this test, generalizations will not be advanced until a matrix of test conditions is investigated.

\footnotetext{
S.D.K. Barbat is associated with the University of Michigan, Depart ment of Mechanical Engineering and Applied Mechanics, Ann Arbor, MI. Y. Guo (SEM Member) is associated with Virginia Polytechnic Institute and State University, Department of Engineering Science and Mechanics, Blacksburg, VA. R.S. Hao is associated with the University of Michigan, Department of Mechanical Engineering and Applied Mechanics, Ann Arbor, MI. D. Post (SEM Member) is associated with Virginia Polytechnic Institute and State University, Department of Engineering Science and Mechanics, Blacksburg, VA.
}

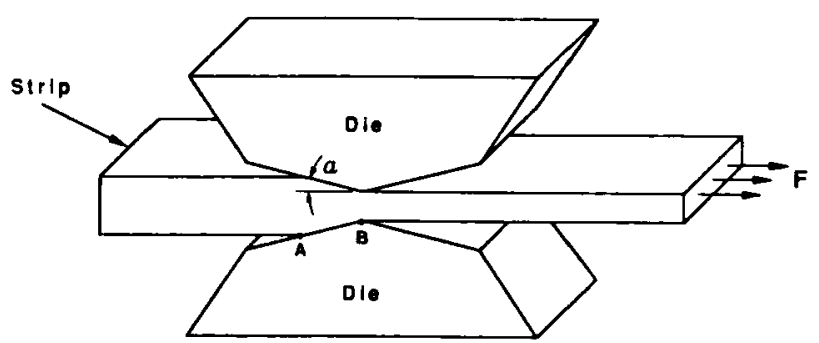

Fig. 1-Schematic diagram of strip drawing process. $A B$ is the contact zone, where $A$ is the entrance point and $B$ is the exit point

\section{THE TEST}

The dies were mounted in a robust test fixture which was secured to the movable cross-head of a screw-driven testing machine. The steel strip, or workpiece, had a reduced thickness section so it could be slipped between the dies prior to drawing. The exit end of the strip was clamped to the fixed head of the testing machine. No lubricant was used between the dies and the workpiece. The cross section of the workpiece was initially $25.4 \times 0.785 \mathrm{~mm}$ and it was reduced in thickness by 22 percent to $0.612 \mathrm{~mm}$. The workpiece width did not change in the drawing operation. The drawing speed, i.e., the cross-head speed, was $1.3 \mathrm{~mm} / \mathrm{min}$.

The optical apparatus for moiré interferometry was also installed on the movable cross head of the testing machine, as illustrated in Fig. 2. The $U$ and $V$ displacement fields of the external surface of the die were obtained by means of a three-mirror system [see Ref. 3, p. 345]. A high-frequency crossed-line specimen grating, with its lines oriented parallel and perpendicular to the contact surface, was previously replicated on the die [Ref. 3, p. 341]. The incident collimated beam was formed by a parabolic mirror and an optical fiber, with the fiber end located at the focal plane of the mirror; both elements were mounted on the testing machine. Light input to the fiber was from an argon-ion laser operating at 514-nm wavelength and 50-mW power.

A $35-\mathrm{mm}$ single-lens reflex camera was used with a $90-\mathrm{mm}$ focal length macroscopic lens, adjusted for slightly less than unit magnification. The film was Kodak Technical Pan and development was by Kodak D-19. Exposure time was 1/1000 sec. 


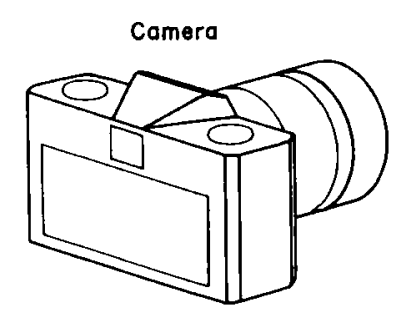

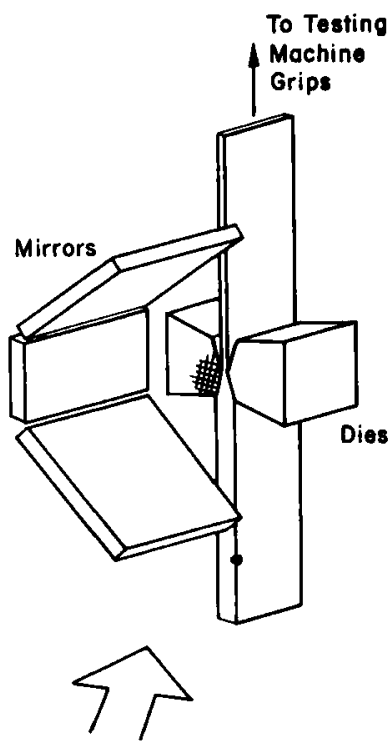

Beam of Collimated Laser Light

Fig. 2-Schematic diagram of the apparatus mounted on the testing machine

The initial frequency of the specimen grating was $f / 2=$ 1200 lines $/ \mathrm{mm}$. The virtual reference grating frequency was $f=2400$ lines/mm and the corresponding sensitivity of the displacement measurements was $1 / f=0.417 \mu \mathrm{m} / \mathrm{fringe}$ order. By visual fringe interpolation, the displacement resolution was about $0.1 \mu \mathrm{m}$.

\section{RESULTS}

The fringe patterns depicting the $U$ and $V$ displacement fields (i.e., contour maps of the $x$ and $y$ components of displacement, respectively) are shown in Fig. 3. Since the $x y$ coordinate directions are aligned with respect to the contact surface of the die, $\sigma_{y}$ and $r_{x y}$ represent normal and tangential boundary conditions in the contact zone. Although the direction of drawing was vertical in the actual test, the results are shown for horizontal drawing, conforming to Fig. 1.

The numbers marked on the patterns are fringe orders $N_{x}$ and $N_{y}$, where

$$
U=\frac{1}{f} N_{x} \quad V=\frac{1}{f} N_{y}
$$
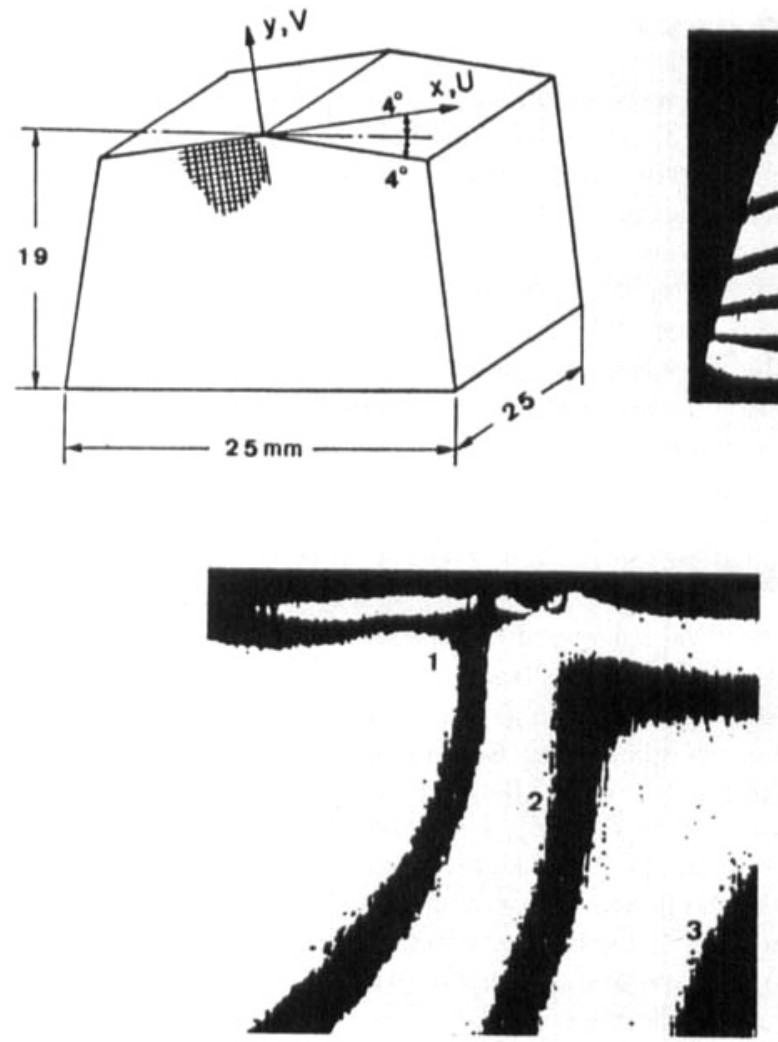

$\mathrm{U}$

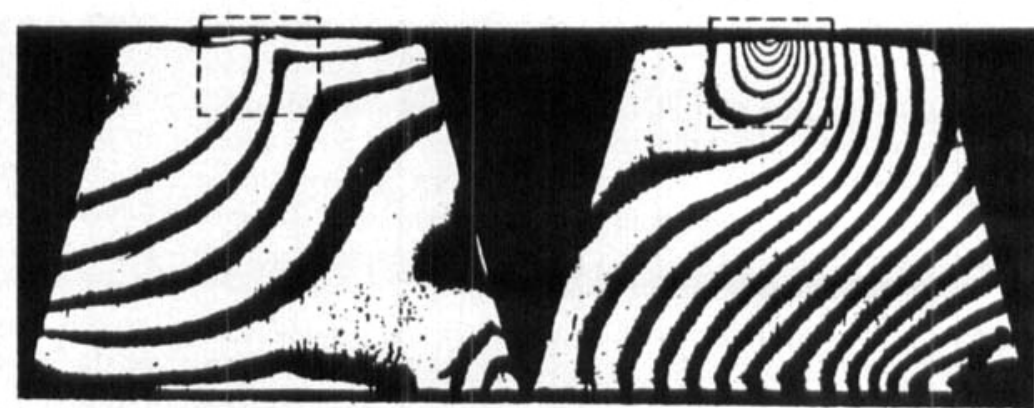

U

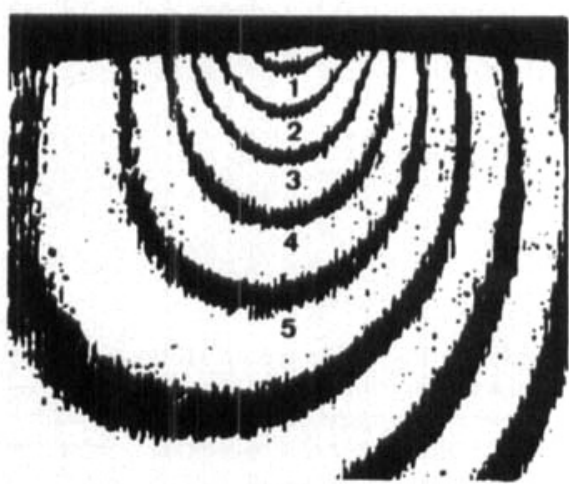

V

Fig. 3-Fringe patterns of the $U$ and $V$ displacement fields for the entire die, with enlargements for the contact region. The numbers, which apply to adjacent black fringes, are fringe orders $N_{x}$ and $N_{y}$ in the $U$ and $V$ fields, respectively 
The location of the zero-order fringe is arbitrary [Ref. 3, p. 347] since rigid-body motions are inconsequential, but the signs of fringe gradients, i.e., the ascending or descending orders of neighboring fringes are assigned authentically.

Strains were determined from the fringe gradients by

$$
\begin{gathered}
\epsilon_{x}=\frac{\partial U}{\partial x}=\frac{1}{f} \frac{\partial N_{x}}{\partial x} \quad \epsilon_{y}=\frac{\partial V}{\partial y}=\frac{1}{f} \frac{\partial N_{y}}{\partial y} \\
\gamma_{x y}=\frac{\partial U}{\partial y}+\frac{\partial V}{\partial x}=\frac{1}{f}\left[\frac{\partial N_{x}}{\partial y}+\frac{\partial N_{y}}{\partial x}\right]
\end{gathered}
$$

and stresses were determined by the small-strain relation ships

$$
\begin{gathered}
\sigma_{y}=\frac{E}{1-\nu^{2}}\left(\epsilon_{y}+\nu \epsilon_{x}\right) \\
\tau_{x y}=G \gamma_{x y}
\end{gathered}
$$

where $E$ and $G$ are the modulus of elasticity and shear modulus of the die material, respectively, and $\nu$ is its Poisson's ratio.

The strains and stresses in the contact zone of the die boundary were determined accordingly. An important result is plotted in Fig. 4, which shows the ratio of shear to normal stresses along the contact boundary. The most interesting feature is the change of sign. While the normal stress was compressive (i.e., negative) along the entire contact length, the shear stress changed from negative near the entrance of the die to positive near the exit. The tangential shear stress $\tau_{x y}$ existed because of frictional forces between the workpiece and the die. The results reveal that frictional forces in the exit region act in the direction of drawing, but in the reverse direction in the entrance region.

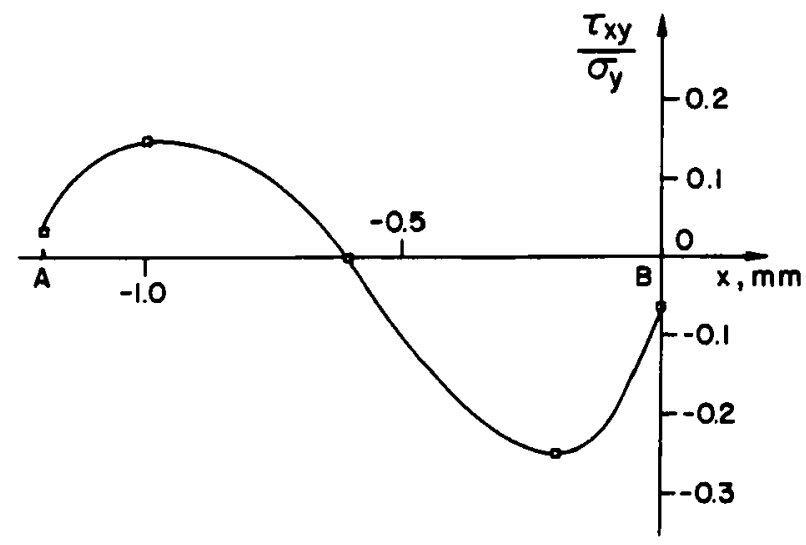

Fig. 4-The ratio of shear stress to normal stress along the contact length

In this test there were only a few fringes in the contact region and the fringe gradients were too small to determine their magnitudes with high accuracy. This issue will be addressed in future work. Nevertheless, the signs of the fringe gradients and the essential nature of their variation appear to be well established. The conclusions to be drawn here rely on the trends of Fig. 4 rather than the numerical magnitudes.

\section{IMPLICATIONB, CONGLUSIONE}

If relative motion between the workpiece and die existed along the contact interface, then $\tau_{x y} / \sigma_{y}$ would represent the coefficient of kinetic friction. The coefficient cannot be zero, and therefore kinetic conditions could not exist in the central part of the contact length. In fact, the kinetic coefficient of friction for steel on steel (without lubricant) is expected to exceed all the measured values of $\tau_{x y} / \sigma_{y}$. This indicates that conditions of static friction existed along the entire contact length, i.e., the relative motion was zero. Under such circumstances the movement along the interface is arrested and deformation continues by subsurface flow, or shear, in the workpiece material. In terms more familiar to the mechanics of fluid flow, stagnation occurred at the contact boundary, signifying an immobile boundary layer in the workpiece.

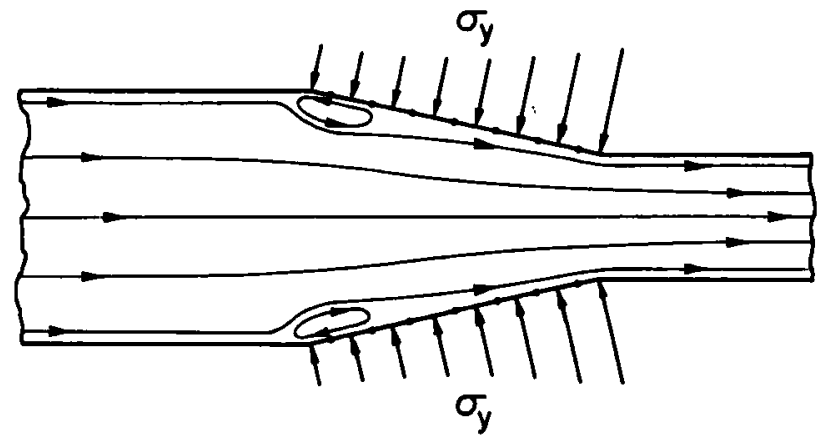

Fig. 5-Schematic diagram of streamlines of metal flow in a non-lubricated drawing process. The dots signify a static boundary layer and the external arrows denote the strong compressive forces induced by the die

Interior to the boundary, the direction of metal flow must correspond to the directions of shear stresses on the boundary. This means a flow reversal occurred near the entrance region. The particle flow is rationalized to follow the streamlines sketched in Fig. 5. A local circulation is generated near the entrance zone, which under steadystate conditions has neither an inflow nor outflow of material. Since this is a case of viscous flow with particle velocity gradients normal to the flow, shear stresses are induced in the material, including the shear at the static interface. Accordingly, the circulation accounts for the reverse direction of frictional forces on the die. The static boundary layer and reverse flow are the dramatic conclusions. 


\section{FUTURE WORK}

Refinements and extensions should address improved accuracy and diverse drawing conditions. Accuracy would be enhanced by a larger contact zone, which would produce more displacement fringes in the contact region. This calls for increased workpiece thickness, since the contact length is proportional to workpiece thickness if the percent reduction is held constant. In addition, accuracy should be enhanced by the use of carrier fringes, ${ }^{4}$ which augment the resolution of fringe gradient determinations.

Future research should investigate the drawing process with lubricated dies. Other variables should include workpiece material, percent reduction, drawing speed, die material and die shape. The objective should be to establish the phenomenological nature of the process in order to guide the formulation of theoretical models and to obtain accurate numerical results to evaluate the validity of proposed models. In the event that theoretical models cannot be sufficiently general, the research should provide numerical data to support hybrid experimental/theoretical analyses of the drawing process, wherein numerical analyses are augmented by experimentally-determined boundary conditions.
Two-dimensional flow is an important assumption made in the analysis. The two-dimensionality should be investigated in future work by microscopic inspection of plastic flow lines, after appropriate polishing and etching of successive cross sections.

\section{ACKNOWLEDGMENT'S}

Support by the National Science Foundation under research grant DMC-8521744 is greatly appreciated.

\section{REFERENGES}

1. Rao, R.S., Devenpeck, M.L., Wright, P.K., Appleby, E.J., Lu, C.Y. and Richmond, O., "New Strip Drawing Experiments Using Trans parent Dies," Int. J. Mech. Sci, 27, (11\& 12), 725.740 (1985).

2. Barbat, S.D.K., Lee, H.Y. and Rao, R.S., "Experimental Procedures for Measuring Boundary Conditions in Forming Processes," Proc. of ASME Annual Meeting, Chicago, IL (1988).

3. Post, D., "Moiré Interferometry," Cha. 7, Handbook of Experimental Mechanics, A.S. Kobayashi, Editor, Prentice-Hall, Englewood Cliffs, NJ (1987).

4. Guo, Y., Post, D. and Czarnek, R., 'The Magic of Carrier Fringes in Moiré Interferometry," EXPERIMENTAL MECHANICS (to be published).

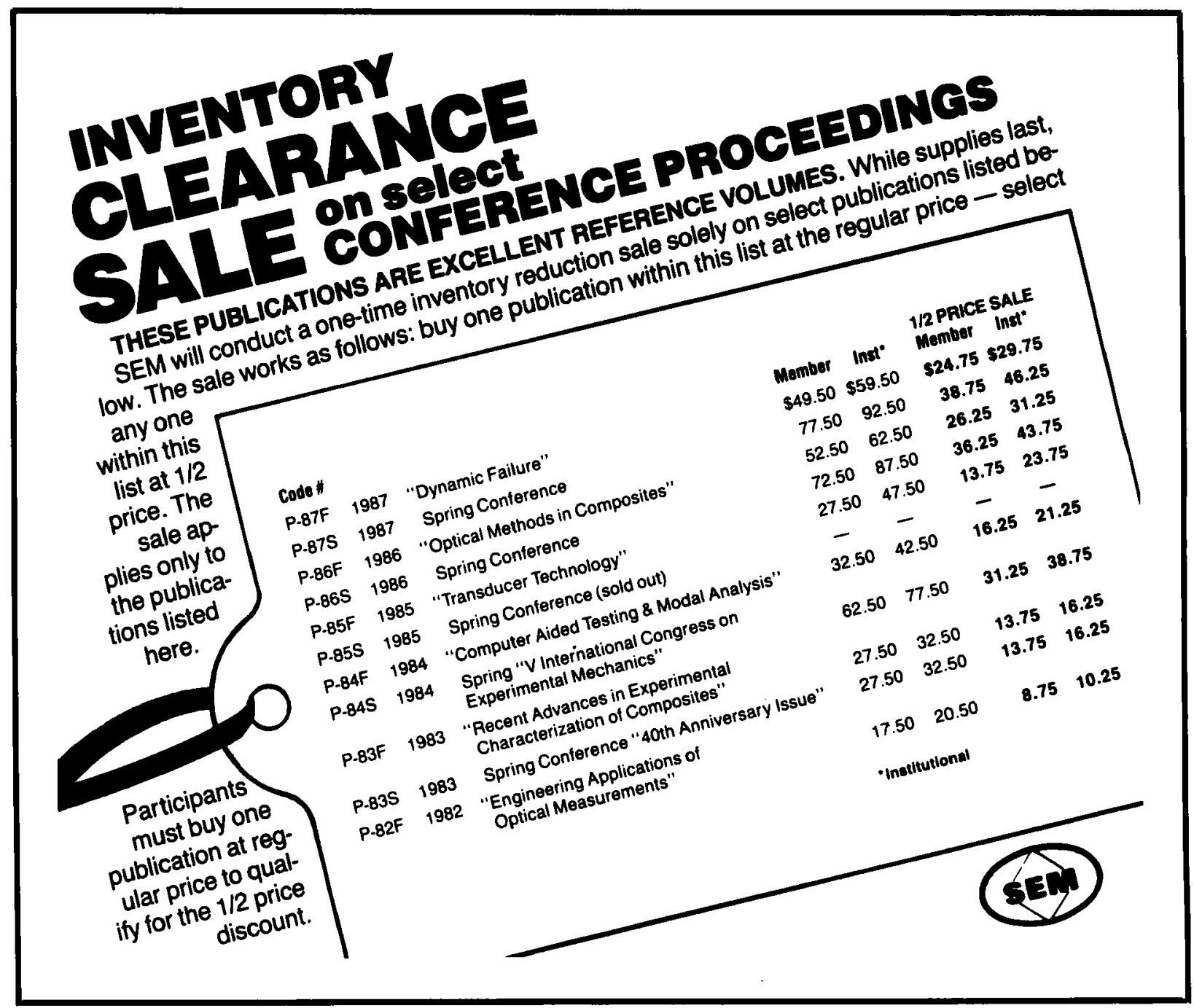

